\title{
DESIGUALDAD, CRISIS FINANCIERA Y ESTANCAMIENTO: HISTORIAS ALTERNATIVAS Y POR QUÉ IMPORTAN
}

Thomas I. Palley*

$\mathrm{E}$ ste artículo examina las historias alternativas del papel de la desigualdad del ingreso en la crisis financiera de 2008 y el estancamiento posterior. A un nivel, es un ejercicio puramente analítico. A otro nivel, hay un propósito más profundo, el de criticar el monopolio neoclásico en economía que ha destruido el pluralismo y distorsionado el debate económico y el diseño de políticas.

En una economía pluralista de mente abierta deben estar representadas todas las teorías que dan una explicación lógicamente coherente de la economía consistente con los hechos que conocemos. Pero en la disciplina no sucede así debido al monopolio neoclásico.

El pluralismo no es solo importante como aspiración intelectual. Lo es también en términos prácticos para proponer una política económica sólida. La teoría da forma a nuestra comprensión del mundo, y esta influye en nuestra respuesta a los hechos. La teoría es una manera de contar historias, y las historias que contamos dan forma a nuestra comprensión de la economía y la política económica. Esto significa que las historias que contamos son esenciales.

El artículo examina algunas explicaciones convencionales de la crisis financiera y del estancamiento y el papel que atribuyen a la

\footnotetext{
* Doctor en Economía. AFL-CIO, USA, [mail@thomaspalley.com]. Este escrito se presentó originalmente en el Foro del Diálogo Europeo 2015, organizado por la Fundación Hans Böckler, celebrado en Bruselas, Bélgica, 16-17 de abril de 2015. Tomado de Real-World Economics Review (RWER) 74, 7 de abril 2016, pp. 1-18. La traducción, de Alberto Supelano, se publica con las autorizaciones correspondientes. Fecha de recepción: 15 de abril de 2016, fecha de aceptación: 12 de mayo de 2016. Sugerencia de citación: Palley, T. I. "Desigualdad, crisis financiera y estancamiento: historias alternativas y por qué importan", Revista de Economia Institucional 18, 34, 2016, pp. 45-68. DOI: http://dx.doi.org/10.18601/01245996. v18n34.05
} 
desigualdad del ingreso. Después se contrastan con la explicación keynesiana estructural ${ }^{1}$. El papel de la desigualdad del ingreso es sustancialmente diferente en esas explicaciones y da lugar a recomendaciones de política diferentes. Eso ilustra la importancia de las historias teóricas que contamos sobre la economía. Por ello es esencial que contemos la historia correcta.

\section{LÍNEAS DE FALLA: RAJAN (2010)}

Rajan (2010) fue uno de los primeros en contribuir a la nueva oleada de reflexiones que atribuyen un papel a la desigualdad en la crisis financiera. Según él, la creciente desigualdad del ingreso en Estados Unidos provocó una respuesta política populista para hacer más asequible la propiedad de vivienda. Ello implicó una intervención del gobierno en el mercado financiero de vivienda que alentó la propiedad de vivienda más allá de los medios personales y provocó una burbuja de crédito impulsada por el precio de la vivienda. Cuando la burbuja estalló en 2006, la estructura financiera de apoyo se derrumbó.

Cabe destacar tres características de esta historia. Primera, la afirmación de que la crisis financiera de 2008 fue causada por la intervención del gobierno en el mercado de vivienda es infundada empíricamente (Palley, 2012, cap. 6). Esa intervención tuvo lugar durante varias décadas. La Ley de Reinversión en la Comunidad se

1 La crisis financiera de 2008 y la Gran Recesión subsiguiente despertaron nuevo interés en los efectos de la desigualdad del ingreso entre los economistas convencionales. Los economistas no tradicionales prestaron atención a la desigualdad durante más de tres décadas antes de la crisis. En el plano teórico, el enfoque keynesiano se basa en el modelo de crecimiento neokaleckiano propuesto por Rowthorn (1982) y refinado por Bhaduri y Marglin (1990) para incluir la distinción entre crecimiento impulsado por los salarios e impulsado por las ganancias. Stockhammer (2011) revisa dicha literatura. Palley (2011) presenta un marco de política para alentar el crecimiento impulsado por los salarios.

E1 trabajo empírico no convencional que documenta el aumento de la desigualdad del ingreso incluye: Galbraith (1998) y la bienal El Estado del trabajador estadounidense producida por Larry Mishel y sus coautores del Instituto de Política Económica de Washington desde 1986. E1 trabajo analítico no convencional sobre el impacto económico de la desigualdad y su tendencia a generar estancamiento incluye: Peterson (1994), Palley (1998), Stanford (1999), Pollin (2003) y Glyn (2006). El interés de los académicos convencionales fue suscitado inicialmente por la investigación empírica de Piketty y Saez (2003) y Gordon y Dew-Becker (2008). Ese interés se ha vuelto viral desde la publicación de El capital en el siglo $X X I$, de Piketty (2014). E1 nuevo interés convencional en la política es evidente en el discurso sobre la desigualdad del ingreso de Janet Yellen, presidente de la Reserva Federal (octubre de 2014), y en su llamado a hacer más investigación sobre los efectos de la desigualdad (abril de 2015). También es evidente en los documentos de investigación de alto perfil del FMI sobre crecimiento, redistribución y desigualdad (Berg y Ostry, 2011; Ostry et al., 2014) y sobre sindicatos y desigualdad (Jaumotte y Buitron, 2015). 
aprobó en 1977 y la Asociación Federal Hipotecaria Nacional (FNMA o Fannie Mae) se fundó en 1938 durante el New Deal. Los préstamos subprime, que desataron la crisis, fueron originados por prestamistas privados y Fannie Mae solo empezó a comprarlos y a facilitar su emisión cerca del final de la burbuja. Por último, la burbuja de precios impactó con igual fuerza los bienes raíces comerciales, pero estos no estaban sujetos a la intervención del gobierno.

Segunda, según Rajan el mercado de trabajo funcionaba de manera eficiente y la distribución del ingreso no era un problema microeconómico ni macroeconómico. Era, en cambio, económicamente justificada por los desarrollos técnicos que habían aumentado el rendimiento del trabajo calificado y disminuido el del trabajo no calificado, y solo era un problema porque provocó una política defectuosa motivada políticamente. Por tanto, aunque aborda el tema de la desigualdad del ingreso, Rajan se aparta del razonamiento que sostiene que esta desigualdad genera problemas de demanda agregada y es el resultado del desigual poder de negociación en el mercado de trabajo. Si no se lee con cuidado, es muy fácil atribuir erróneamente este argumento a Rajan, cuando en realidad no lo menciona en su libro.

Tercera, su libro no considera las implicaciones referentes al estancamiento. Para explicarlo, después argumentó que los efectos posteriores a las crisis económicas asociadas al alto apalancamiento son muy duraderos (Rajan y Ramcharan, 2015). Eso lo pone en compañía de Reinhart y Rogoff (2009), pero su inferencia empírica de largas recesiones posteriores a las crisis financieras es cuestionada por Christina y David Romer (2015), quienes encuentran que cuando los trastornos financieros se clasifican en una escala relativamente fina, en vez de ser tratados como una variable $0-1$, "la reducción del producto después de las crisis financieras en los países avanzados es muy variable, en promedio moderada y a menudo transitoria”.

\section{"DESIGUALDAD, APALANCAMIENTO Y CRISIS": KUMHOFY RANCIÈRE (2010)}

Una segunda contribución al debate sobre el papel de la desigualdad del ingreso en la crisis proviene de Kumhof y Rancière (2010). Su explicación es una mezcla de teoría keynesiana de la demanda y de teoría clásica de la oferta. $\mathrm{El}$ argumento es que un empeoramiento de la distribución del ingreso, causado por el descenso del poder sindical de negociación, produjo un ascenso persistente del endeudamiento cuando los trabajadores intentaron mantener su nivel de vida. Eso llevó a que la economía fuera financieramente frágil y vulnerable a otro 
choque sobre el poder de negociación de los trabajadores que redujo aún más su ingreso, de modo que no pudieron pagar sus préstamos.

Pero un examen más atento muestra que la historia es mucho menos keynesiana de lo que parece. En primer lugar, la economía es una economía de pleno empleo antes y después de la crisis, de modo que la distribución del ingreso nada tiene que ver con el pleno empleo. En segundo lugar, el papel de la distribución del ingreso es impulsar el endeudamiento que ocasiona la fragilidad financiera. Eso significa que su explicación de la crisis es en realidad una explicación de fallas del mercado financiero en forma de préstamos excesivos que lleva a que la economía sea vulnerable a choques. Sin endeudamiento excesivo, el deterioro de la distribución del ingreso no es un problema, excepto por razones éticas.

En tercer lugar, según la historia de Kumhof y Rancière la crisis financiera fue precedida por otro choque adverso sobre el poder de negociación de los trabajadores que redujo sus ingresos y entonces no pudieron pagar sus préstamos. Pero no hay pruebas de tal choque en 2006-2007. Por el contrario, ese fue un periodo de pleno empleo relativo en el que aumentó el poder de negociación de los trabajadores, como lo evidencia el aumento de los salarios reales.

Por último, el modelo tiene dificultades para explicar el tamaño de la reducción del producto causada por la crisis financiera y por qué el estancamiento cristalizó después de la Gran Recesión. La explicación de Kumhof y Rancière supone que la crisis financiera destruyó el 10\% del acervo de capital, lo que no es plausible.

\section{"DEUDA, DESAPALANCAMIENTO Y TRAMPA DE LIQUIDEZ": EGGERTSSON Y KRUGMAN (2012)}

Una tercera explicación del estancamiento es el conjunto de argumentos asociados con la trampa de la tasa de interés nominal con límite inferior cero (LIC). Quien dio origen a este marco fue Paul Krugman (1998), que lo desarrolló originalmente para explicar el estancamiento de Japón después del colapso de su burbuja de precios de los activos en 1991. Eggertsson y Krugman (2012) refinaron la historia para intentar explicar el estancamiento posterior a la crisis financiera estadounidense de 2008.

La historia que precede al estancamiento es que una burbuja financiera impulsó un endeudamiento y un apalancamiento excesivos en la economía de Estados Unidos. Cuando la burbuja explotó en 2007-2008, la economía sufrió una crisis financiera y una recesión profunda. También provocó una oleada de desapalancamiento cuando los 
prestatarios pasaron a reconstruir sus balances. E1 desapalancamiento aumentó el ahorro que la economía ha sido incapaz de absorber debido a la LIC. E1 exceso de ahorro resultante redujo la demanda agregada y con ello causó el estancamiento.

Esta historia del estancamiento se describe en la gráfica 1. El quid de la historia es la pretensión de que existe una tasa de interés que produce pleno empleo, y que la tasa de interés necesaria se determina en el mercado de fondos prestables mediante la oferta de ahorro y la demanda de inversión. El papel de la tasa de interés es equilibrar el ahorro de pleno empleo y la inversión de pleno empleo ${ }^{2}$. El desapalancamiento aumenta el ahorro y ocasiona un desplazamiento hacia afuera de la curva de oferta de ahorro de pleno empleo, de modo que la igualación entre el ahorro y la inversión de pleno empleo requiere una tasa real de interés negativa. Sin embargo, debido a la LIC la tasa de interés nominal no puede ser negativa. En consecuencia, hay un exceso de oferta de ahorro que provoca una contracción del ingreso y del empleo.

\section{Gráfica 1}

La explicación del estancamiento basada en el desapalancamiento

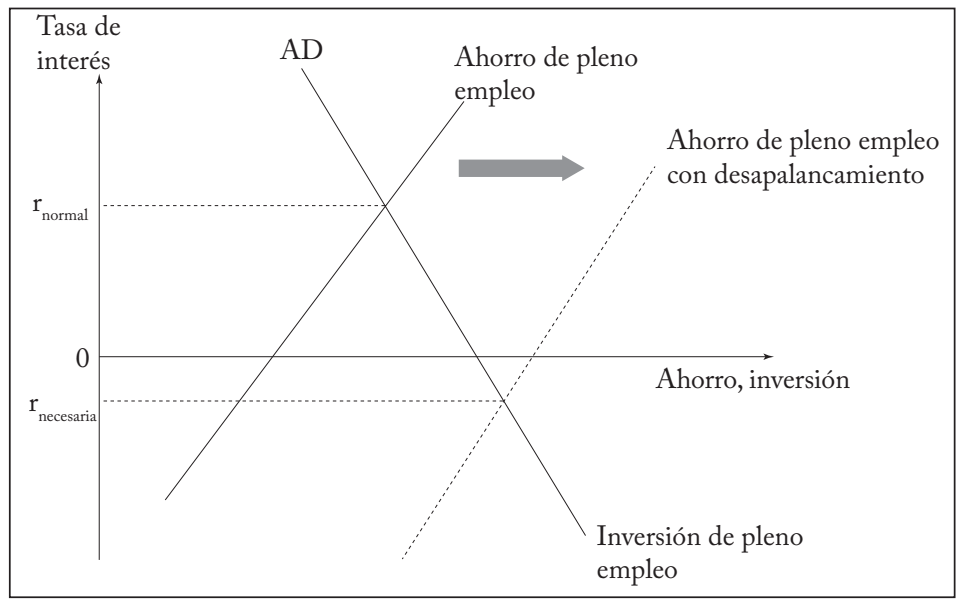

La solución de política tiene dos aspectos: 1) generar grandes déficits presupuestales para que el déficit del sector público absorba el exceso de ahorro del sector privado; 2) alentar las expectativas de inflación para que la tasa real de interés esperada sea negativa aunque la tasa de interés nominal de mercado esté atrapada en cero. Hay varias características de la historia LIC que son problemáticas. En primer

${ }^{2}$ En el modelo Krugman-Eggertsson el Banco Central logra esta tasa de interés de pleno empleo mediante sus metas de tasas de interés. 
lugar y al nivel más general, se sustenta en una teoría de las tasas de interés basada en los fondos prestables, en la cual la tasa de interés es determinada por la oferta de ahorro y la demanda de inversión. Ese enfoque fue desprestigiado hace mucho tiempo por Keynes en su Teoria general.

En segundo lugar, atribuye demasiada importancia a las tasas de interés como origen del problema y como medio para resolver los problemas de empleo e inestabilidad de una economía capitalista. Se pretende que una tasa de interés real negativa del $3 \%$ aumentaría $\mathrm{AD}$ hasta restaurar el pleno empleo. No obstante, las tasas de interés real fueron negativas en los años setenta y eso no resolvió los problemas de empleo de esa época. Hoy, una tasa de interés negativa del $3 \%$ podría desencadenar una nueva burbuja financiera que estallaría en forma peor una vez las tasas reales de interés empezaran a revertirse hacia arriba. Esa inconsistencia sugiere que hay un problema más profundo en la economía que la historia LIC de Eggertsson-Krugman (2012) no puede identificar.

En tercer lugar, la historia del desapalancamiento basada en el exceso de ahorro y la escasez de demanda no es convincente. En efecto, como muestra el cuadro 1, la deuda de las empresas no financieras de Estados Unidos ha aumentado muy rápidamente desde 2011. Y la deuda de los hogares se contrajo poco durante la Gran Recesión y también ha aumentado desde 2012. Además, es probable que gran parte de la reducción de la deuda de los hogares proviniera de incumplimientos y deudas condonadas, lo que probablemente aumenta la demanda agregada y reduce el ahorro aliviando las obligaciones de los deudores.

Cuadro 1

Crecimiento de la deuda de los hogares y las empresas no financieras Estados Unidos

\begin{tabular}{lccccccc}
\hline & 2008 & 2009 & 2010 & 2011 & 2012 & 2013 & 2014 \\
\hline Hogares & 1,1 & 0,0 & $-1,1$ & $-0,2$ & 1,5 & 1,5 & 2,9 \\
Empresas & 5,8 & $-4,3$ & $-0,9$ & 3,0 & 4,8 & 5,1 & 5,9 \\
\hline
\end{tabular}

Fuente: Cuentas financieras de Estados Unidos, Reserva Federal, cuarto trimestre de 2014.

En cuarto lugar, en realidad la explicación Eggertsson-Krugman del estancamiento no atribuye ningún papel a la desigualdad del ingreso. La distribución del ingreso se puede añadir a la historia suponiendo que los hogares de ingresos más altos tienen mayor propensión a ahorrar $^{3}$. En ese caso, un desplazamiento de la distribución del ingreso

3 Palley (2010) presenta una justificación teórica integral de las diferencias en la propensión a consumir de los hogares deudores y acreedores. La teoría es 
hacia hogares de ingresos más altos aumentaría el ahorro de pleno empleo. En términos de la gráfica 1, tendría un efecto idéntico al del desapalancamiento y desplazaría a la derecha la función de ahorro de pleno empleo. Sin embargo, aunque esto añade efectos de distribución del ingreso al modelo de Eggertsson-Krugman, no resuelve las otras críticas referentes a la lógica económica y la importancia del razonamiento LIC. Para explicar el estancamiento es necesario añadir la distribución del ingreso, pero se debe añadir a otra historia.

\section{LA IMPORTANCIA ECONÓMICA DE LA DESIGUALDAD PARA EL ESTANCAMIENTO}

Además de introducir la LIC como explicación del estancamiento, Krugman ha impugnado continuamente la importancia de la desigualdad para explicar el estancamiento:

Joe Stiglitz argumenta en Opinionator que la desigualdad es un factor impor-
tante en nuestra lenta recuperación. Joe es un grandioso economista y todo
lo que dice se debería tomar en serio. Y en vista de mis opiniones políticas
y mi preocupación general por la desigualdad, me gustaría estar de acuerdo.
Pero -se sabía que seguía un "pero"- he pensado mucho en esos temas, y
no he podido convencerme de que este cuento moral particular sea cierto
(Krugman, 2013a).

La esencia de su rechazo de la importancia económica de la desigualdad es que el ahorro privado estadounidense como proporción del PIB disminuyó en los años anteriores a la crisis financiera, pese a que la desigualdad estaba aumentando. Como muestra la gráfica 2, la tasa de ahorro declinó notablemente desde 1980 hasta 2000, lo que supuestamente prueba que la desigualdad no reduce la demanda:

Observe el ahorro privado total como proporción del PIB: la tendencia antes de la crisis era hacia abajo, no hacia arriba; y es claro que el ascenso durante la crisis no fue impulsado por un aumento de la desigualdad. ¿Digo por ello que se puede tener pleno empleo con base en la compra de yates, carros lujosos y servicios de entrenadores personales y chefs célebres? Bien, sí. No tiene por qué gustarle, pero la economía no es un juego moral (Krugman, 2013a).

¿Qué es erróneo en este argumento de que una tasa de ahorro en descenso que muestra una creciente desigualdad del ingreso no provoca escasez de demanda? El problema es que no tiene en cuenta otros desarrollos que contrarrestaban y ocultaban los efectos adversos de

consistente con los hechos estilizados establecidos del gasto de consumo: que la propensión agregada a consumir de largo plazo es mayor que la propensión a corto plazo (Kuznets, 1946); la observación de corte transversal de que los hogares de ingresos más altos tienen una mayor propensión a ahorrar (Carroll, 2000); y la observación de corte transversal de que la varianza del ingreso de los hogares es mayor que la varianza de su consumo (Krueger y Perri, 2002). 
demanda del empeoramiento de la distribución del ingreso, como se ilustra en la gráfica 3. La época neoliberal empezó formalmente con la llegada del presidente Reagan (en realidad, ya estaba en marcha con el presidente Carter, que inició la desregulación y nombró a Paul Volcker con el mandato de reducir la inflación con altas tasas de interés). Como argumentó Palley (2012), el paso a la política neoliberal provocó dos cambios fundamentales. El primero fue una época de estancamiento de los salarios y de ampliación de la desigualdad del ingreso. El segundo fue una época de inflación de precios de los activos y de una burbuja de crédito de treinta años de duración que aumentó la riqueza, las garantías, el volumen de crédito y la facilidad para obtener crédito. Esos desarrollos financieros que alentaron el gasto compensaron con creces los efectos negativos del estancamiento de los salarios, y explican por qué cayó la tasa de ahorro aunque la desigualdad del ingreso estuviese aumentando. La burbuja de crédito terminó con la crisis financiera, que puso fin a una época de endeudamiento estrafalario. Eso llevó a una recuperación de la tasa de ahorro que provocó una escasez de demanda. Esta explicación, que se ajusta a los hechos descritos en el cuadro 1 y la gráfica 2, muestra que el aumento del ahorro causado por la desigualdad del ingreso, y no por el desapalancamiento, es responsable del estancamiento.

Gráfica 2

Ahorro del sector privado como proporción del PIB Estados Unidos, 1947-2012

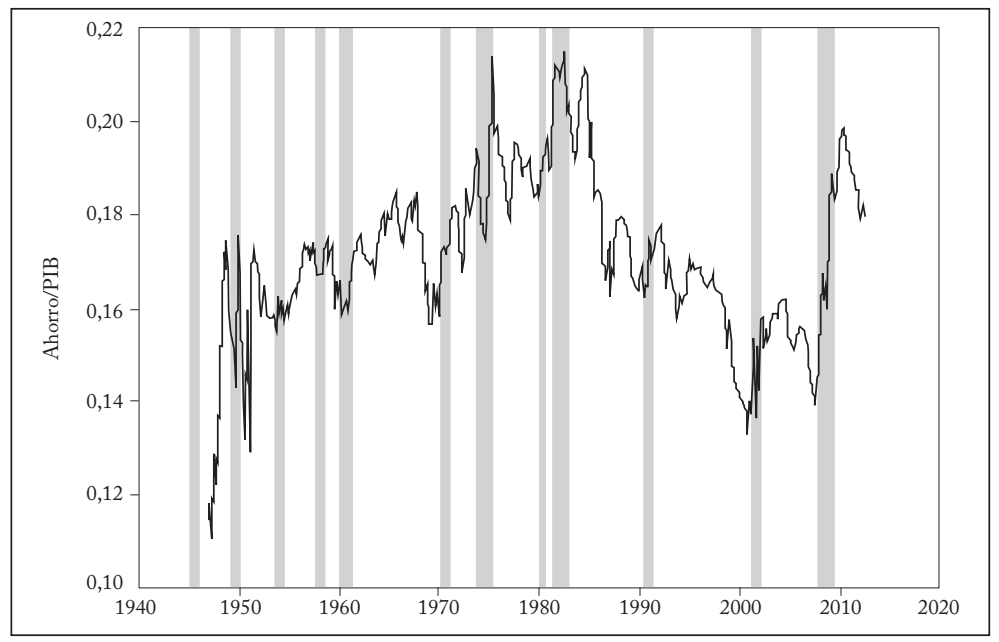

Nota: las áreas sombreadas indican recesiones en Estados Unidos.

Fuente: Federal Reserve Economic Data (FRED). 
Cynamon y Fazzari (2014) presentan sólida evidencia que respalda esta secuencia de hechos. Reportan que el aumento del ingreso del $95 \%$ inferior de los hogares se estancó antes de 2006, pero la proporción entre su ingreso y su deuda se elevó a niveles insostenibles. Desde la Gran Recesión esa relación deuda-crédito bajó a niveles más sostenibles mediante el proceso de impago de la deuda, restricción del acceso al crédito y aceptación por los hogares de que la inflación futura del precio de los activos no cancelaría las deudas. Sus hallazgos respaldan plenamente la hipótesis de que el endeudamiento ocultó los efectos adversos de demanda de la desigualdad antes de la crisis, y que el rezago de demanda de la desigualdad salió a la superficie cuando la orgía de endeudamiento llegó a su fin.

Gráfica 3

Evolución de la economía estadounidense en la época neoliberal, 1980-2015

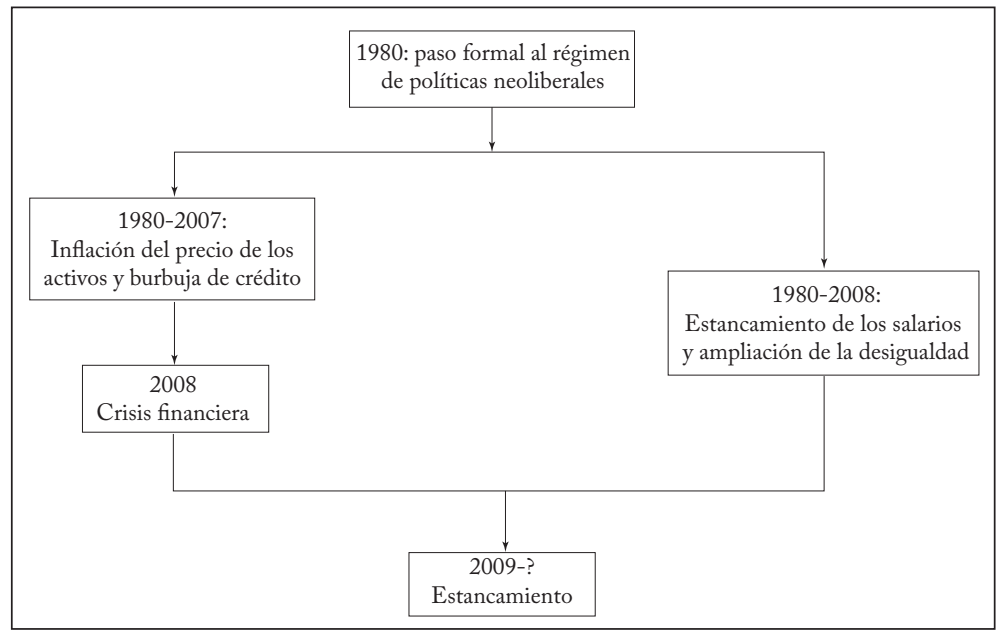

\section{LA EXPLICACIÓN KEYNESIANA ESTRUCTURAL DE LA DESIGUALDAD Y EL ESTANCAMIENTO: PALLEY (2009; 2012)}

$\mathrm{E} 1$ argumento anterior muestra que la distribución del ingreso importa, y que se debe incorporar en una historia macroeconómica mejor que la de la LIC. Esta sección presenta una explicación "keynesiana estructural" de la crisis financiera y el estancamiento (Palley, 2009; 2012), escrita mucho antes de que economistas tradicionales como Larry Summers percibieran el estancamiento. Por ello, esta explicación es muy inusual debido a que anticipó correctamente los desarrollos inminentes. 
La explicación es la siguiente. Hasta finales de la década de 1970 las economías de los países desarrollados, incluida la de Estados Unidos, se podían describir mediante un modelo de crecimiento keynesiano de círculo virtuoso en el que los salarios eran el motor del crecimiento de la demanda. La lógica económica se ilustra en la gráfica 4 . El crecimiento de la productividad impulsó el crecimiento de los salarios, el cual alimentó el crecimiento de la demanda. Eso promovió el pleno empleo que proporcionó el incentivo para invertir, lo que elevó aún más el crecimiento de la productividad. En este sistema, las finanzas eran caracterizadas por un modelo de utilidad pública basado en la regulación del New Deal. Su papel era proporcionar: (a) fondos de inversión a las empresas y a los empresarios; (b) servicios de seguros a las empresas y a los hogares, y (c) medios de ahorro a los hogares para necesidades futuras.

Gráfica 4

El modelo de crecimiento keynesiano de círculo virtuoso, 1945-1975

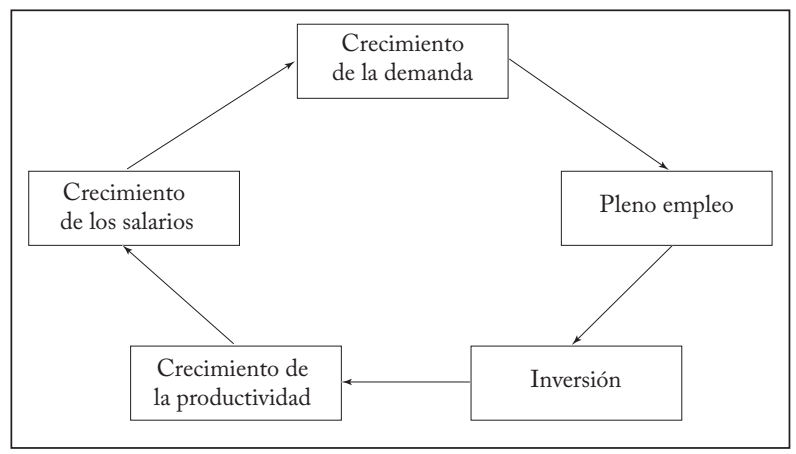

Después de 1980 el modelo de crecimiento keynesiano de círculo virtuoso fue remplazado por un modelo de crecimiento neoliberal. Los dos cambios clave en la economía real fueron: 1) el abandono de la política de compromiso con el pleno empleo, que fue sustituido por un compromiso con una inflación estable baja, y 2) el corte del vínculo entre crecimiento de los salarios y de la productividad. Además, hubo un cambio en el sector financiero impulsado por la “financiarización", que aumentó la presencia y el poder de las finanzas en la economía. En conjunto, estos cambios crearon un nuevo modelo económico. Antes de 1980, los salarios eran el motor de crecimiento de la demanda; después de 1980, los motores fueron la deuda y la inflación del precio de los activos. 
Como muestra la gráfica 5, el nuevo modelo económico se puede describir como una "caja de política neoliberal" que encierra a los trabajadores y los presiona por todos lados mediante (1) el modelo de globalización corporativo; (2) la agenda de un gobierno pequeño, que ataca a la regulación y a la actividad del sector público; (3) la agenda de flexibilización del mercado de trabajo, que ataca a los sindicatos, al poder de negociación de los trabajadores y a su protección, y (4) la sustitución de la política macroeconómica de pleno empleo por la política cuya meta es una baja inflación. En cuanto al sistema financiero, el modelo de utilidad pública del New Deal fue destrozado por la desregulación y las innovaciones financieras que quedaron desreguladas. El resultado fue un nuevo sistema caracterizado por una creciente inestabilidad financiera, el estancamiento de los salarios y el aumento de la desigualdad del ingreso.

Gráfica 5

La caja neoliberal

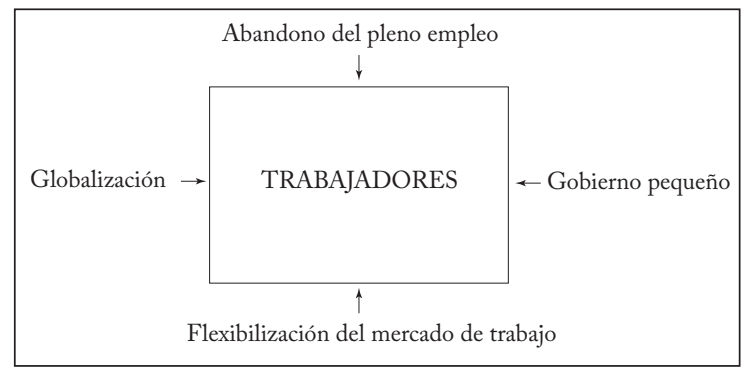

Estos desarrollos de los salarios y del ingreso provocaron una creciente escasez estructural de demanda. El papel de las finanzas fue llenar esa brecha. La desregulación financiera, la innovación financiera, la especulación y el fraude financiero de viejo tipo hicieron posible que las finanzas llenaran la brecha de demanda dando crédito a los consumidores e inflando los precios de los activos.

Cabe señalar algunas características. Primera, que las finanzas llenaran esta "brecha de demanda" no era parte de un plan grandioso: fue una consecuencia inesperada. Los diseñadores de las políticas neoliberales no se dieron cuenta de que estaban generando una brecha de demanda, pero su ideología financiera de laissez-faire provocó desarrollos que la llenaron accidentalmente. Segunda, el proceso era inevitablemente inestable y siempre estuvo destinado a la implosión. Hay límites para el endeudamiento y para la inflación de precios de los activos. Todo esquema Ponzi se derrumba eventualmente. E1 
problema es que es imposible predecir cuándo sucederá. Tercera, el proceso fue de larga duración. Por ello el derrumbe fue mucho más profundo cuando ocurrió. Esto también implica que librarse de los efectos es mucho más difícil porque ahora la economía está agobiada por las deudas y se ha destruido la solvencia crediticia.

\section{LA VISIÓN KEYNESIANA ESTRUCTURAL DEL PAPEL DE LA DESIGUALDAD EN LA CRISIS Y EL ESTANCAMIENTO}

La explicación keynesiana estructural de los hechos tiene diferencias sutiles con las explicaciones populares. La desigualdad del ingreso no causó la crisis financiera. La crisis fue causada por la implosión del precio de los activos y de las burbujas de crédito que habían compensado y ocultado el impacto de la desigualdad. Pero una vez la burbuja financiera estalló y los mercados financieros dejaron de llenar la brecha de demanda creada por la desigualdad del ingreso, los efectos de demanda de la desigualdad pasaron a primer plano. Visto a esa luz, el estancamiento es el producto conjunto de la prolongada burbuja de crédito, la crisis financiera y la desigualdad del ingreso. La burbuja de crédito dejó una gran deuda pendiente, la crisis financiera destruyó la solvencia crediticia de millones de personas y la desigualdad del ingreso generó una escasez de demanda "estructural".

Este diagnóstico también aclara por qué el pronóstico de mediano plazo sigue siendo el estancamiento. Porque la política no ha resuelto estos problemas fundamentales y en realidad los ha empeorado. En primer lugar, Estados Unidos aún tiene una "brecha de demanda" estructural ocasionada por el deterioro de la distribución del ingreso, la cual ha empeorado desde la crisis de 2008. En segundo lugar, la burbuja de crédito se acabó y el endeudamiento ya no puede llenar la "brecha de demanda". Además, las reformas del sector financiero han endurecido sistemáticamente el acceso al crédito. En tercer lugar, las fugas de importaciones e inversión asociadas a la globalización aún no se han reparado, mientras que el estímulo fiscal ha recurrido a la austeridad fiscal. En consecuencia, a pesar de las políticas de la Reserva Federal de tasa de interés cero y flexibilización cuantitativa (FC), la economía está asediada por un crecimiento más lento y la flexibilización general del mercado de trabajo será cada vez mayor. Además, existe el peligro de que los precios de los activos se vuelvan a inflar y de que el experimento de FC sea contraproducente y provoque nuevos trastornos en el mercado financiero. 


\section{LA HISTORIA QUE ACEPTAMOS IMPORTA}

En las secciones anteriores se resumieron cuatro historias diferentes sobre el papel de la desigualdad del ingreso en la crisis financiera y el estancamiento. Cuál historia aceptemos es de suma importancia porque la manera de explicar el mundo afecta la manera de entenderlo, la que a su vez tiene delicadas consecuencias políticas y de política.

Si se acepta la historia de Rajan (2010) la distribución del ingreso se reduce a un tema de preocupación política y ética, pero no es un asunto de preocupación macroeconómica. Además, puesto que los mercados de trabajo funcionan tal como se supone, no hay justificación para que se intervengan con el fin de aumentar la participación de los salarios o fortalecer el poder de negociación de los trabajadores. En vez de centrarse en la desigualdad del ingreso, la respuesta de política debería ser abolir la intervención del gobierno en la financiación de vivienda y retornar a una política monetaria más ortodoxa para evitar la posibilidad de otra burbuja de precios de los activos. También puede haber argumentos para una redistribución del ingreso después de impuestos, pero ese es un asunto puramente ético y político.

Si se acepta la historia de Kumhof y Rancière (2010), la causa de la crisis es la falla del mercado financiero que permitió el endeudamiento excesivo de los hogares trabajadores cuyas perspectivas de ingreso habían disminuido. La respuesta de política debería ser endurecer la regulación del mercado financiero para prevenir la repetición de una burbuja de endeudamiento endeble. Pero, de nuevo, los mercados de trabajo están funcionando eficientemente. Eso significa que la redistribución del ingreso para aumentar la participación de los salarios es, de nuevo, puramente ética y política.

Si se acepta la historia LIC del desapalancamiento, de Eggertsson-Krugman, la distribución del ingreso también se reduce a un asunto no económico. La causa del estancamiento es, en cambio, el desapalancamiento, un proceso que se debe remediar. Sin embargo, hay pruebas de que durante este periodo los grandes déficits presupuestales compensaron el exceso de ahorro privado causado por el desapalancamiento, y evitaron cualquier pérdida de producto y empleo causada por la obstrucción LIC al pleno empleo. Puesto que el mercado de trabajo es eficiente y no la causa del problema, esto significa que la distribución del ingreso es, una vez más, un asunto puramente ético y político, y que no hay razones económicas para la intervención encaminada a aumentar la participación de los salarios.

Si se acepta la historia "keynesiana estructural", la distribución del ingreso es un problema central y el factor determinante de la escasez 
de demanda que causa el estancamiento. La solución es remplazar el marco neoliberal de política por un marco "keynesiano estructural”. Metafóricamente hablando, se necesita que los diseñadores de política rearmen la caja, saquen de allí a los trabajadores, y pongan dentro a las corporaciones y a los mercados financieros. Como ilustra la gráfica 6, eso requiere sustituir la globalización corporativa por una globalización administrada; restablecer el compromiso de la política macroeconómica con el pleno empleo; remplazar la agenda anti estatal por una agenda socialdemócrata que apoye y financie la inversión pública, la provisión de servicios públicos y la regulación (incluida la de los mercados financieros); y cambiar la flexibilización neoliberal del mercado de trabajo por la solidaridad basada en mercados de trabajo donde los trabajadores tengan mayor poder de negociación y reciban una mayor participación de los salarios.

Gráfica 6

Rearmar la caja

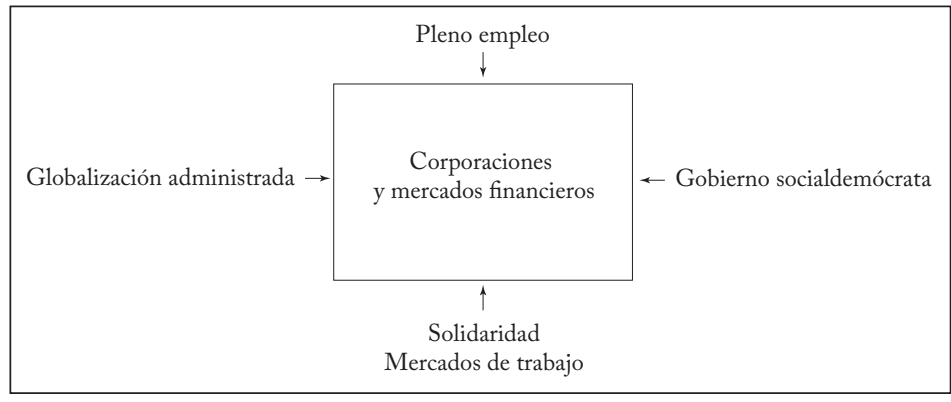

Sin embargo, hay una capa de complejidad adicional asociada a la financiarización, que hace diferente la economía política actual de la del pasado. Rearmar la caja de política económica exige recobrar el control sobre los intereses financieros que han cumplido un papel esencial en la creación y el mantenimiento del nuevo modelo económico. Este papel de las finanzas se ilustra en la gráfica 7. En primer lugar, las finanzas usaron su poder político para promover las políticas en las que descansa el nuevo modelo. Si se araña cualquier lado de la caja neoliberal de política queda visible la influencia de las finanzas. Cabildearon por la desregulación financiera; apoyaron el cambio de la política macroeconómica de centrarse en el pleno empleo para centrarse en la inflación; respaldaron la globalización corporativa y la ampliación de la movilidad internacional del capital; apoyaron la privatización, la agenda de impuestos regresivos y la reducción del 
Estado; y secundaron el ataque a los sindicatos y al trabajo encaminado a reducir los salarios.

Gráfica 7

Los principales canales de la financiarización

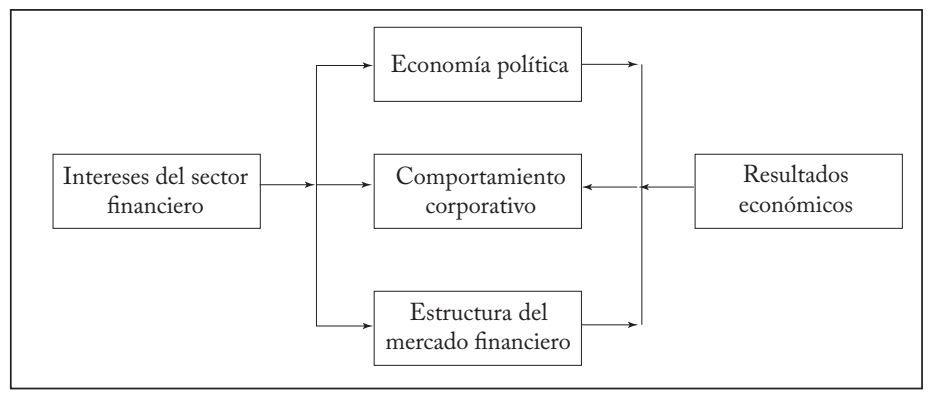

En segundo lugar, las finanzas tomaron control de las empresas y las indujeron a adoptar puntos de vista y comportamientos del sector financiero. El cambio se justificó con el argumento de maximizar el valor de los accionistas. El resultado fue la adopción del modelo de compras apalancadas que cargó de deudas a las firmas, de un esquema de negocios de corto plazo, de tasas de rendimiento requeridas excesivamente altas que debilitan la inversión de largo plazo, del apoyo a la deslocalización y el abandono del compromiso con las comunidades; y de la adopción de paquetes de remuneración similares a los de Wall Street para directores y altos ejecutivos.

En tercer lugar, los mercados financieros desregulados y las innovaciones financieras proporcionaron el crédito para financiar compras apalancadas, absorciones y recompras de acciones. También respaldaron el crédito hipotecario y de consumo que infló el precio de la vivienda y llenó temporalmente la "escasez de demanda" creada por el estancamiento de los salarios. Las finanzas cubrieron la brecha de demanda creada por el modelo neoliberal de política, pero el costo fue la creación de una estructura financiera cada vez más frágil que colapsó con la crisis de 2008.

E1 esbozo de un programa para recobrar el control de las finanzas podría ser el siguiente (Palley, 2014). Una reforma política y electoral que reduzca el papel del dinero privado; un cambio de política monetaria para dar más peso al pleno empleo que a la inflación; una reforma de la gobernanza corporativa que desaliente la perspectiva gerencial de corto plazo de maximizar el precio de las acciones, y una reforma de la regulación financiera que permita usar la política 
cuantitativa para manejar el tamaño y la composición de los balances de las empresas financieras.

\section{LA DESIGUALDAD Y LAS FALLAS DE POLÍTICA ECONÓMICA COMO CAUSAS DEL ESTANCAMIENTO}

Hasta aquí la atención se ha centrado en el papel económico de la desigualdad en la generación del estancamiento. La economía política señala otro canal a través del cual la desigualdad afecta la política económica. Por cierto, Krugman (2013b) argumenta que la economía política ha sido el canal principal. Su argumento es que la desigualdad creciente aumentó el poder político de los ricos, que favorecieron las políticas de austeridad fiscal que causaron el estancamiento:

\footnotetext{
En mi opinión, sin embargo, el papel realmente crucial de la desigualdad en la calamidad económica ha sido político. En los años anteriores a la crisis había en Washington un notable consenso bipartidista en favor de la desregulación financiera; un consenso que no estaba justificado por la teoría ni por la historia. Cuando estalló la crisis se corrió a rescatar a los bancos. Pero después de rescatarlos surgió un nuevo consenso, que implicó alejarse de la creación de empleo y centrarse en la supuesta amenaza de los déficits presupuestales [...] Las encuestas a los muy ricos muestran, sin embargo, que -a diferencia del público en general- ellos consideran que los déficits presupuestales son un tema crucial y están a favor de grandes recortes de los programas de seguridad social. $\mathrm{Y}$, por supuesto, esas prioridades se impusieron en nuestro discurso político (Krugman, 2013b).
}

En opinión de Krugman, el estancamiento es el resultado de la falla en el uso de la política fiscal con el fin de compensar el desapalancamiento, y esa falla de política se puede atribuir a los efectos políticos del aumento de la desigualdad del ingreso.

Cabe señalar algunos puntos importantes. Primero, este argumento de economía política es totalmente consistente con la hipótesis keynesiana estructural. De hecho, Palley $(2012,205-207)$ argumenta explícitamente que el poder y la riqueza han moldeado las ideas económicas que alientan la política neoliberal. El incremento de la desigualdad del ingreso solo ha reforzado esa influencia.

Segundo, aunque sin intención, el argumento de Krugman va al meollo del debate. Para él, no hay nada "estructuralmente" erróneo en la economía. Esta pasa por un proceso de desapalancamiento que se debe corregir, y el estímulo fiscal puede ayudar a corregirlo con más rapidez y menos dolor. En cambio, en la hipótesis keynesiana estructural el origen del estancamiento es la defectuosa estructura de la economía. La austeridad fiscal agravó el estancamiento, pero no es la causa esencial. 
Tercero, la visión de Krugman y la visión keynesiana estructural comparten la idea de que la política económica es la causa del estancamiento. Pero igual que en el debate sobre el impacto económico de la desigualdad del ingreso, es importante acoger la historia correcta sobre el papel de la política económica. Para Krugman (2013b), la falla de política es el giro a la austeridad fiscal después de 2009. Esto contrasta con la hipótesis keynesiana estructural que localiza la falla de política a finales de la década de 1970 y el paso a políticas neoliberales. Esa es una historia muy distinta y tiene implicaciones de política muy diferentes. Muestra, una vez más, la importancia de acoger la historia correcta.

\section{LA RESISTENCIA DE LATEORÍA TRADICIONAL A LA DESIGUALDAD}

Rajan, Kumhof, Rancière y Krugman son grandes economistas tradicionales. Han estado vinculados a la Universidad de Chicago, al FMI y al MIT. En términos de inclinación intelectual, Rajan se identifica con el neoliberalismo duro de la escuela de Chicago que considera la economía como un sistema semejante al modelo de competencia perfecta de los libros de texto. Argumenta que las fallas de mercado son raras y relativamente pequeñas. Además, así no sean pequeñas, la intervención del gobierno para corregirlas produce resultados aún peores porque está sujeta a fallas del gobierno, que son mucho más costosas que las fallas del mercado.

Kumhof, Rancière y Krugman se identifican con el neoliberalismo blando de la escuela del MIT. Creen en el mismo marco de referencia del modelo de competencia perfecta de los neoliberales duros. Pero argumentan que las fallas de mercado son ubicuas y grandes, y que se requiere la política del gobierno para hacer la buena tarea de remediar sus efectos.

La relación entre neoliberalismo duro y blando se muestra en la gráfica 8. El punto importante es que Rajan, Kumhof, Rancière y Krugman comparten la visión teórica convencional de la economía; aunque difieran en la extensión de las fallas de mercado y en la efectividad de la intervención correctiva del gobierno. Esa visión contrasta notablemente con la visión keynesiana estructural.

Este contraste es particularmente agudo en lo que respecta a la desigualdad. La economía convencional tiene profunda resistencia a reconocer sus impactos de eficiencia, quizá porque la desigualdad es el tema políticamente más contencioso. Reconocer sus impactos de eficiencia daría razones convincentes para remediarla, lo que implicaría desafiar el statu quo y los intereses pecuniarios de la élite. 
Gráfica 8

La estructura intelectual de la economía moderna

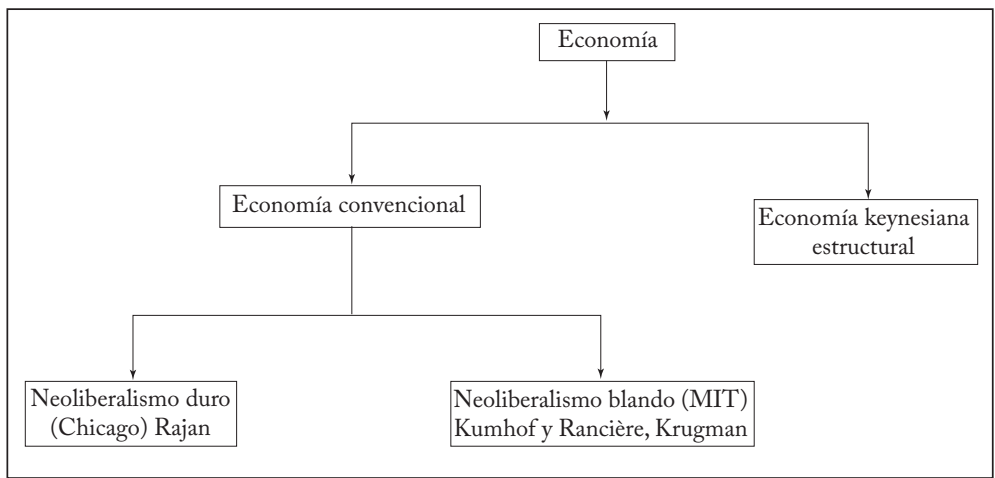

Una causa de la resistencia a reconocer los efectos macroeconómicos de eficiencia de la desigualdad es el modelo de equilibrio general competitivo de Arrow-Debreu (1954), que sigue siendo el núcleo analítico de la economía teórica convencional. Ese modelo describe una economía “ideal" y genera los dos famosos teoremas del bienestar. El primero afirma que las economías perfectamente competitivas, sin fallas de mercado o de información, generan resultados de equilibrio óptimos de Pareto. Esas economías son eficientes en materia de producción y asignación, en el sentido de que ninguna persona puede estar mejor haciendo que otra esté peor, y este resultado es válido independientemente de cuán igual o desigual sea la distribución inicial de la riqueza.

El segundo teorema afirma que en una economía ideal la única manera de redistribuir la riqueza y el ingreso sin generar ineficiencias de producción ni de asignación son los impuestos de suma fija. Puesto que tales impuestos son imposibles en el mundo real, este teorema hace imposible corregir la desigualdad sin incurrir en pérdidas de eficiencia.

Estos dos teoremas solo son válidos en esa economía ideal, pero marcan el pensamiento económico convencional de una manera que produce dos sesgos. Primero, la desigualdad no importa para la eficiencia económica. Segundo, es probable que corregir la desigualdad aumente la ineficiencia económica.

Una segunda causa de resistencia intelectual es la teoría neoclásica del comportamiento microeconómico que trasmite una disposición favorable hacia la desigualdad. Esa disposición es captada por Arthur Okun, un importante economista liberal del pasado, que escribió: 
Los contrastes en el nivel de vida y la riqueza material de los hogares estadounidenses reflejan un sistema de premios y castigos que busca promover el esfuerzo y canalizarlo hacia la actividad socialmente productiva. Pero esa búsqueda de eficiencia necesariamente crea desigualdades. Y, por tanto, la sociedad enfrenta un trade-off entre igualdad y eficiencia (Okun, 1975, 1).

Este argumento de los incentivos se ha infiltrado profundamente en la economía y en el pensamiento social, que aceptan la pretensión de Okun de un gran trade-off entre igualdad y eficiencia.

Una tercera causa de resistencia e indiferencia intelectual hacia la desigualdad proviene de la macroeconomía y las teorías convencionales del consumo. Según la hipótesis del ingreso permanente (Friedman, 1957), todos los individuos tienen la misma propensión marginal a consumir, lo que hace irrelevantes la distribución y la desigualdad del ingreso para la demanda agregada. Según la teoría del consumo del ciclo biológico (Modigliani y Brumberg, 1954), la propensión a consumir depende de la edad del individuo. La distribución por edades y la distribución del ingreso entre hogares de edades diferentes es lo que importa para el consumo agregado, y no la distribución del ingreso per se.

La combinación de estos argumentos -teoría del equilibrio general competitivo de Arrow-Debreu, teoría neoclásica de incentivos microeconómicos y teoría macroeconómica del consumo- ha contribuido a que los economistas convencionales sean indiferentes e incluso apoyen la desigualdad. Y ayuda a explicar por qué está tan ausente en sus explicaciones del estancamiento. En cambio, la economía keynesiana tiene una perspectiva muy diferente, en la que la desigualdad puede ser una causa importante de ineficiencia macroeconómica.

$\mathrm{E} 1$ argumento keynesiano empieza con el rechazo de la economía ideal de Arrow-Debreu y su pretensión de pleno empleo. En vez de ella, describe la economía del mundo real como una economía monetaria marcada por una incertidumbre fundamental acerca del futuro, en la cual la demanda agregada puede disminuir cuando las personas retardan sus planes de gasto en respuesta a la incertidumbre. Además, el sistema de mercado puede ser incapaz de restaurar el nivel de demanda agregada lo suficiente para asegurar el pleno empleo debido a que los bajos precios y la deflación aumentan la carga de la deuda, alientan a las personas a retrasar aún más el gasto e inducen incumplimientos de pagos que pueden desestabilizar el sistema bancario y trastornar los mercados financieros.

De acuerdo con la economía keynesiana, la demanda agregada es el determinante decisivo de la actividad económica. Además, el gasto de consumo es afectado por la desigualdad (Palley, 2010) pues los 
hogares más ricos tienen una propensión al ahorro más alta que los más pobres. En consecuencia, el aumento de la desigualdad puede incrementar el ahorro y reducir la demanda agregada, provocando un desempleo keynesiano que el mercado no puede remediar.

En cuanto a la teoría microeconómica de los incentivos, las motivaciones del comportamiento son mucho más variadas y maleables de lo que sugiere Okun $(1975,1)$. Su visión refleja un punto de vista estadounidense. En una sociedad donde el dinero es la métrica dominante de la autovaloración y la autoestima, es posible que los incentivos monetarios sean mucho más potentes. Pero también es posible que en otras sociedades sean más importantes otras métricas de valor y autoestima, y menos potentes los incentivos monetarios. Lo cual implica que lo que nos motiva se construye socialmente y cuestiona profundamente la visión de un agudo y difícil trade-offentre eficiencia y desigualdad.

Además, el carácter de los arreglos de incentivos permitidos también importa enormemente. Los torneos en que el ganador se toma todo son una motivación poderosa, especialmente en una sociedad donde el dinero es la métrica del valor y la protección social es débil. Pero pueden ser socialmente subóptimos porque las reglas del torneo son determinadas por los propietarios que recaudan el excedente del torneo, y los trabajadores que participan pueden preferir otros arreglos de incentivos. Esa es la lección de la economía de la competencia de ratas que genera una horrible carrera hacia abajo (Akerlof, 1976; Palley, 1998, 9).

En suma, la visión keynesiana estructural de la desigualdad es esencialmente diferente de la visión convencional. La desigualdad es una causa de fallas de demanda agregada y promueve sistemas de incentivos que pueden ser socialmente subóptimos. Sin embargo, el monopolio académico de la economía neoliberal de Chicago y del MIT impide que esa visión tenga audiencia.

\section{CONCLUSIÓN: OTRA VEZ LA ECONOMÍA DEL GATOPARDO}

Hay tres conclusiones principales. Primera, las cuatro historias anteriores se asemejan en cuanto mencionan "la distribución del ingreso" o "la escasez de demanda", pero son esencialmente diferentes. Si los lectores no agudizan su ingenio, es fácil que pasen por alto esas diferencias fundamentales.

La posibilidad de confusión aumenta porque las distintas historias pueden llevar a un traslapo de las recomendaciones de política. Por ejemplo, la historia LIC de Krugman recomienda usar estímulos 
fiscales, igual que la historia keynesiana estructural. Pero estas dos historias difieren esencialmente en su explicación de las causas de la crisis y del estancamiento. Eso suscita un asunto crítico. No basta encontrar puntos de acuerdo en la política: también es necesario obtener la historia correcta de la economía. Una historia equivocada induce a los diseñadores de políticas y al público a errores sobre cómo pensar la economía, promueve una respuesta incompleta de política, y suscita futuros desacuerdos analíticos y de política.

Segunda, existe el gran peligro de la "economía gatopardo" (Palley, 2013), un cambio que no cambia a la economía. Durante treinta años, los keynesianos progresistas han sostenido que la distribución del ingreso tiene importancia macroeconómica. Hoy los economistas tradicionales están entendiendo este asunto. E1 peligro del gatopardo es que la incorporen en sus historias de una manera que despoje a la distribución del ingreso de su importancia esencial para la eficiencia macroeconómica y que así desmonten el argumento en favor de la intervención de política para reducir la desigualdad del ingreso.

Tercera, el artículo describió cuatro historias. Tres de ellas son muy citadas y conocidas. Se enseñan en las escuelas de postgrado y se discuten en el FMI y en los bancos centrales. La cuarta (la historia keynesiana estructural) está proscrita a un agujero negro. No por falta de evidencia o de lógica. De hecho, su lógica y su evidencia son superiores. Está proscrita debido, en cambio, al "poder de los intereses", que asegura que solo ciertas ideas se discutan en las aulas y en el debate público. Esos intereses incluyen los de los ricos, pero también los de la profesión, que está organizada como un club y solo da voz a las ideas de los miembros de su club.

Estas conclusiones tienen importantes implicaciones prácticas. Dada la importancia vital de "obtener la historia correcta", la acción progresista encaminada a modificar la política debe ir acompañada de vigorosos esfuerzos para cuestionar y sustituir la historia económica convencional. La modificación de la historia es un proyecto que tiene dos aspectos: 1) requiere difundir la explicación keynesiana estructural alternativa de la crisis y del estancamiento, y 2) implica cuestionar la teoría económica convencional que constituye el fundamento del neoliberalismo duro y blando. En ausencia de una modificación de la historia económica, es improbable que los progresistas triunfen en el debate político sobre las políticas y arreglos económicos necesarios para la prosperidad compartida y la buena sociedad. 
Esa falla es visible en la evolución política posterior a la crisis financiera de 2008. Con el fracaso para modificar la historia, la política económica retornó a los lugares comunes anteriores: austeridad fiscal, flexibilidad del mercado laboral y más globalización corporativa. Solo la política monetaria mantiene una modalidad diferente, pero también amenaza volver a la modalidad anterior a la crisis con el primer soplo de inflación. En cuanto a la política electoral, en Estados Unidos el partido republicano ha obtenido grandes ganancias políticas, en el Reino Unido el partido conservador derrotó al partido laborista, y en Alemania los demócratas cristianos conservadores derrotaron a los socialdemócratas. En parte, esa evolución política refleja la falla para entender la historia correcta y presentar al electorado una narración keynesiana estructural alternativa claramente definida.

\section{REFERENCIAS BIBLIOGRÁFICAS}

1. Akerlof, G. "The economics of caste and of the rat race and other woeful tales", Quarterly Journal of Economics 90, 4, 1976, pp. 599-617.

2. Arrow, K. J. y G. Debreu. "Existence of an equilibrium for a competitive economy", Econometrica 22, 3, 1954, pp. 265-290.

3. Berg, A. y J. D. Ostry. "Inequality and unsustainable growth: Two sides of the same coin?", IMF staff discussion note, abril de 2011.

4. Bhaduri, A. y S. A. Marglin. "Unemployment and the real wage: The economic basis for contesting political ideologies", Cambridge Journal of Economics 14, 4, 1990, pp. 375-393.

5. Carroll, C. D. "Why do the rich save so much?", J. Slemrod, ed., Does Atlas shrug? The economic consequences of taxing the rich, Cambridge, Mass., Harvard University Press, 2000.

6. Cynamon, B. Z. y S. M. Fazzari. "Inequality, the great recession and slow recovery", 2014, [http://papers.ssrn.com/sol3/papers.cfm?abstract id $=2205524]$.

7. Eggertsson, G. B. y P. Krugman. "Debt, deleveraging, and the liquidity trap: A Fisher-Minsky-Koo approach”, Quarterly Journal of Economics 127, 3, 2012, pp. 1469-1513.

8. Friedman, M. A theory of the consumption function, Princeton, Princeton University Press, 1957.

9. Galbraith, J. K. Created unequal: The crisis in American pay, Chicago, University of Chicago Press, 1998.

10. Glyn, A. Capitalism unleashed: Finance, globalization, and welfare, Oxford, UK, Oxford University Press, 2006.

11. Gordon, R. J. e I. Dew-Becker. "Controversies about the rise of American inequality: A survey", NBER working paper No. 13982, mayo de 2008.

12. Jaumotte, F. y C. O. Buitron. "Inequality and labor market institutions", staff discussion note SDN $\backslash 15 \backslash 14$, IMF, julio de 2015.

13. Keynes, J. M. The general theory of employment, interest, and money, Londres, Macmillan, 1936. 
14. Krueger, D. y F. Perri. "Does income inequality lead to consumption inequality? Evidence and theory”, NBER working paper No. 9202, 2002.

15. Krugman, P. "Inequality and recovery", The New York Times, 20 de enero de 2013a.

16. Krugman, P. "Why inequality matters", New York Times, 16 de diciembre, p. A25, 2013b.

17. Krugman, P. "It's baaack: Japan's slump and the return of the liquidity trap", Brookings Papers on Economic Activity 2, 1998, pp. 137-205.

18. Kumhof, M., y R. Rancière. "Inequality, leverage and crises", IMF working paper WP/10/268, Washington DC, 2010.

19. Kuznets, S.; L. Epstein y E. Zenks. National product since 1869, Nueva York, NBER, 1946.

20. Mishel, L. et al. The state of working America, Washington DC, Economic Policy Institute, 1986-2014.

21. Modigliani, F. y R. Brumbergh. "Utility analysis and the consumption function: An interpretation of cross-section data", K. Kurihara, ed., Post Keynesian economics, Londres, Allen and Unwin, 1954.

22. Okun, A. M. Equality and efficiency: The big trade-off, Washington DC, Brookings Institution, 1975.

23. Ostry, J. D.; A. Berg y C. G. Tsangarides. "Redistribution, inequality, and growth", Research Department, IMF, abril de 2014.

24. Palley, T. I. "Making finance serve the real economy", The state of the world 2014, cap. 16, Washington DC, Worldwatch Institute, 2014.

25. Palley, T. I. "Gattopardo economics: The crisis and the mainstream response of change that keeps things the same", European Journal of Economics and Economic Policies 10, 2, 2013, pp. 193-206.

26. Palley, T. I. From financial crisis to stagnation: The destruction of shared prosperity and the role of economics, Cambridge, Mass., Cambridge University Press, 2012.

27. Palley, T. I. "The economics of wage-led recovery: Analysis and policy recommendations”, International Journal of Labor Research 3, 2, 2011, pp. 219-244.

28. Palley, T. I. "The relative permanent income theory of consumption: A synthetic Keynes-Duesenberry-Friedman model”, Review of Political Economy 1, 2010, pp. 41-56.

29. Palley, T. I. "America's exhausted paradigm: Macroeconomic causes of the financial crisis and great recession", New America Foundation, Washington DC, julio de 2009.

30. Palley, T. I. Plenty of nothing: The downsizing of the American dream and the case for structural Keynesianism, Princeton, NJ, Princeton University Press, 1998.

31. Peterson, W. C. Silent depression: Twenty-five years of wage squeeze and middle-class decline, Nueva York, Norton, 1994.

32. Piketty, T. Capital in the Twenty-First Century, Cambridge, Mass., Harvard University Press, 2014.

33. Piketty, T. y E. Saez. "Income inequality in the United States, 19131998", Quarterly Journal of Economics CXVIII, 1, 2003, pp. 1-39.

34. Pollin, R. Contours of descent: US economic fracture and the landscape of global austerity, Londres, Verso, 2003. 
35. Rajan, R. G. Fault lines: How bidden fractures still threaten the world economy, Princeton, Princeton University Press, 2010.

36. Rajan, R. y R. Ramcharan. "The anatomy of a credit crisis: The boom and bust in farm land prices in the United States in the 1920s", American Economic Review 105, 4, 2015, pp. 1439-1477.

37. Reinhart, C. y K. Rogoff. This time is different: Eight centuries of financial folly, Princeton, Princeton University Press, 2009.

38. Romer, C. y D. Romer. "New evidence on the impact of financial crises in advanced countries", NBER working paper No. 21021, marzo de 2015.

39. Rowthorn, R. "Demand, real wages and growth", Studi Economici 19, 1982, pp. 3-54.

40. Stanford, J. Paper boom: Why real prosperity requires a new approach to Canada's economy, Toronto, James Lorimer, 1999.

41. Stockhammer, E. "Wage-led growth: An introduction", International Journal of Labor Research 3, 2, 2011, pp. 167-188.

42. Yellen, J. L. "Perspectives on inequality and opportunity from the Survey of Consumer Finances", Remarks at the Conference on Economic Opportunity and Inequality, Federal Reserve Bank of Boston, Boston, Mass., octubre de 2014.

43. Yellen, J. L. "Opening remarks”, Conference on Economic Mobility, Federal Reserve System, Washington DC, abril de 2015. 